\title{
An Novel Power Flow Calculation Model with the Elimination of Interconnecting Nodes
}

\author{
Shuanbao $\mathrm{Niu}^{1}$, Jili Wang ${ }^{1}$, Chao Huo ${ }^{1}$, and Jun $\mathrm{Liu}^{2^{\star}}$ \\ ${ }^{1}$ Northwest Division of State Grid Corporation of China, Xi'an 710048, China \\ 2 State Key Laboratory of Electrical Insulation and Power Equipment, Xi'an Jiaotong University. \\ Xi'an, 710049, China (Corresponding author, e-mail: eeliujun@mail.xjtu.edu.cn)
}

Keywords: Power flow calculation, interconnecting nodes, computation efficiency.

\begin{abstract}
Plenty of transmission substations in large scale power system are designed to deliver power to electricity users far away from power plants, and most of them can be seen as interconnecting (IC) nodes. Interconnecting node has the characteristic of zero power injection, thereafter causes zero current injection to the power system. A novel power flow calculation model is proposed, based on the elimination of IC node equations, to get more fast power flow calculation. The power network equations of IC nodes are eliminated so as to reduce the total dimension of the power flow model. Then, element-wise Jacobian matrix are deduced according to the reduced system power balance equations, in relationship to partial voltage vectors of non-interconnecting nodes. Tests on IEEE standard systems are then performed to validate the proposed method, and computation efficiency are compared with the traditional formulation of power flow model.
\end{abstract}

\section{Introduction}

Power flow calculation is one of the most fundamental calculation in power system analysis, operation and control, which is a nonlinear power equation solving problem [1]. Traditional power flow calculation aims at computing the nodal voltage amplitude, phase angle under polar coordinate, or in obtaining the real and imaginary parts of the voltage vector under rectangular coordinates. In order to get the power flow solution, Newton-Raphson method is always used for solving linearized power equations [2],[3], and it is able to solve most power flow cases, which has made it the most widely used algorithm in modern power systems.

Since the number of real and reactive power equations is close to twice of the number of buses, the computation time will increase dramatically in large scale power systems. It is well-known that there are plenty of transmission substations in large scale power system, delivering power to electricity users far away from power plants, and most of them can be seen as interconnecting (IC) nodes during power flow calculation. Interconnecting node has the characteristic of zero power injection, thereafter causes zero current injection to the power system. Some optimal power flow applications has introduced current balance equations as equality constraints [4],[5], which divided all buses into two types: the buses with non-zero injections and the buses with zero injections, and makes the total number constraints decrease significantly, which seems very effective for future steady state power flow calculations. Therefore, a new idea of calculating power flow based on the IC nodes reduction technique, seems to be prospective when the power system has a considerable large portion of IC nodes. In fact, large scale power systems with bulk, long distance transmission system substations are mostly the case.

In this paper, a novel power flow calculation model is proposed, based on the elimination of IC node equations, to get more fast power flow calculation. Firstly, the power network equations of IC nodes are eliminated so as to reduce the total dimension of the power flow model in section 2 . Then, element-wise Jacobian matrix are deduced according to the reduced system power balance equations, in relationship to partial voltage vectors of non-interconnecting nodes, in section 3 . Tests on IEEE standard systems are then performed to validate the proposed method in section 4, and computation efficiency are compared with traditional formulation of power flow model. 


\section{New model for power flow calculation}

In an electric power network with $n_{1}$ buses, nodal equation could be expressed according to the following matrix form:

$$
\mathbf{I}=\mathbf{Y V}
$$

Where:

$$
\mathbf{I}=\left[\begin{array}{c}
\dot{I}_{1} \\
\dot{I}_{2} \\
\vdots \\
\dot{I}_{n_{1}}
\end{array}\right], \quad \mathbf{V}=\left[\begin{array}{c}
\dot{V}_{1} \\
\dot{V}_{2} \\
\vdots \\
\dot{V}_{n_{1}}
\end{array}\right], \quad \mathbf{Y}=\left[\begin{array}{cccc}
Y_{11} & Y_{12} & \cdots & Y_{1 n_{1}} \\
Y_{21} & Y_{22} & \cdots & Y_{2 n_{1}} \\
\vdots & \vdots & \ddots & \vdots \\
Y_{n_{1} 1} & Y_{n_{1} 2} & \cdots & Y_{n_{1} n_{1}}
\end{array}\right]
$$

are the nodal injection current vector, and nodal voltage vector, and the node admittance matrix of the network, respectively.

\subsection{Interconnecting node elimination}

Due to the reason that the injection currents for IC nodes are all zero, this part of the network equation can be set apart. Suppose there are $m_{1}$ IC nodes in the power system, and noninterconnecting nodes will be $n=\left(n_{1}-m_{1}\right)$, the network equations can be represented as:

$$
\left[\begin{array}{l}
\mathbf{0} \\
\mathbf{I}_{2}
\end{array}\right]=\left[\begin{array}{ll}
\mathbf{Y}_{11} & \mathbf{Y}_{12} \\
\mathbf{Y}_{21} & \mathbf{Y}_{22}
\end{array}\right]\left[\begin{array}{l}
\mathbf{V}_{1} \\
\mathbf{V}_{2}
\end{array}\right]
$$

The admittance matrix block $\mathbf{Y}_{11}$ is typically non-singular, as the full admittance should be nonsingular so as to get a power flow solution. Then the node voltage block $\mathbf{V}_{1}$ can be eliminated:

$$
\mathbf{V}_{1}=-\left(\mathbf{Y}_{11}\right)^{-1} \mathbf{Y}_{12} \mathbf{V}_{2}
$$

After the elimination of IC nodes, a reduced admittance matrix is then obtained.

$$
\mathbf{Y}_{\text {reduced }}=\mathbf{Y}_{22}-\mathbf{Y}_{21}\left(\mathbf{Y}_{11}\right)^{-1} \mathbf{Y}_{12}
$$

The network equation of Eqn. (3) can be acquired as:

$$
\mathbf{I}_{2}=\mathbf{Y}_{\text {reduced }} \mathbf{V}_{2}
$$

\subsection{Dimension reduction of the power flow model}

Noticing the definition of power system complex power:

$$
\mathbf{S}=\mathbf{V}(\mathbf{I})^{*}
$$

Then the power balance equations of Eqn. (3) can be reduced to a dimension of $n=\left(n_{1}-m_{1}\right)$ as:

$$
\mathbf{S}_{2}=\mathbf{V}_{2}\left(\mathbf{I}_{2}\right)^{*}=\mathbf{V}_{2}\left(\mathbf{Y}_{\text {reduced }} \mathbf{V}_{2}\right)^{*}
$$

Polar coordinate form of node power equations are written as [1]:

$$
\left\{\begin{array}{l}
P_{G i}-P_{L i}-U_{i} \sum_{j=1}^{n} U_{j}\left(G_{i j} \sin \theta_{i j}+B_{i j} \cos \theta_{i j}\right)=0 \\
Q_{G i}-Q_{L i}-U_{i} \sum_{j=1}^{n} U_{j}\left(G_{i j} \sin \theta_{i j}-B_{i j} \cos \theta_{i j}\right)=0
\end{array}\right.
$$

2.3 Matrix form of the dimensional reduced power flow model

The network injection power of Eqn. (9) can be transformed to a matrix form, which is more suitable to be coded in Matlab software:

$$
\begin{aligned}
& U_{i} \sum_{j=1}^{n} U_{j}\left(G_{i j} \cos \theta_{i j}+B_{i j} \sin \theta_{i j}\right) \\
&= {\left[\begin{array}{l}
U_{1} U_{1}\left(G_{11} \cos \theta_{11}+B_{11} \sin \theta_{11}\right)+\cdots+U_{1} U_{n}\left(G_{1 n} \cos \theta_{1 n}+B_{1 n} \sin \theta_{1 n}\right) \\
U_{2} U_{1}\left(G_{21} \cos \theta_{21}+B_{21} \sin \theta_{21}\right)+\cdots+U_{2} U_{n}\left(G_{2 n} \cos \theta_{2 n}+B_{2 n} \sin \theta_{2 n}\right) \\
\cdots \\
U_{n} U_{1}\left(G_{n 1} \cos \theta_{n 1}+B_{n 1} \sin \theta_{n n}\right)+\cdots+U_{n} U_{n}\left(G_{n n} \cos \theta_{n n}+B_{n n} \sin \theta_{n n}\right)
\end{array}\right] } \\
&=\operatorname{diag}[\mathbf{U}][\mathbf{G} \cos \theta+\mathbf{B} \sin \theta][\mathbf{U}] .
\end{aligned}
$$

where: the symbol ${ }^{\operatorname{diag}[]}$ means diagonalization of a column vector. 
The complete power flow model can be obtained as:

$$
\left\{\begin{array}{l}
\mathbf{P}-\operatorname{diag}[\mathbf{U}][\mathbf{G} \cos \boldsymbol{\theta}+\mathbf{B} \sin \boldsymbol{\theta}][\mathbf{U}]=0 \\
\mathbf{Q}-\operatorname{diag}[\mathbf{U}][\mathbf{G} \cos \boldsymbol{\theta}-\mathbf{B} \sin \boldsymbol{\theta}][\mathbf{U}]=0
\end{array}\right.
$$

Using the prosthaphaeresis formula:

$$
\left\{\begin{array}{l}
\cos x \cos y+\sin x \sin y=\cos (x-y) \\
\sin x \cos y-\cos x \sin y=\sin (x-y)
\end{array}\right.
$$

Then the power flow model yields:

$$
\left\{\begin{array}{l}
\mathbf{P}_{i}-\operatorname{diag}[\mathbf{U}]\left[|\mathbf{Y}| \cdot * \cos \left(\boldsymbol{\delta}_{\mathrm{ij}}\right)\right][\mathbf{U}]=0 \\
\mathbf{Q}_{i}-\operatorname{diag}[\mathbf{U}]\left[|\mathbf{Y}| \cdot * \sin \left(\boldsymbol{\delta}_{\mathrm{ij}}\right)\right][\mathbf{U}]=0
\end{array}\right.
$$

In which the element-wise variables can be deducted as : $\operatorname{diag}[\mathbf{U}]\left[|\mathbf{Y}| \cdot * \cos \left(\boldsymbol{\delta}_{\mathrm{ij}}\right)\right][\mathbf{U}]$

$$
=\left(\begin{array}{ccccc}
V_{1} & & & & \\
& \ddots & & & \\
& & V_{i} & & \\
& & \ddots & \\
& & & & V_{n}
\end{array}\right)\left\{\left(\begin{array}{ccccc}
Y_{11} & \cdots & Y_{1 j} & \cdots & Y_{1 n} \\
\vdots & \ddots & \vdots & & \vdots \\
Y_{i 1} & \cdots & Y_{i j} & \cdots & Y_{i n} \\
\vdots & & \vdots & \ddots & \vdots \\
Y_{n 1} & \cdots & Y_{n j} & \cdots & Y_{n n}
\end{array}\right) \bullet \cos \left(\begin{array}{ccccc}
\delta_{11} & \cdots & \delta_{1 j} & \cdots & \delta_{1 n} \\
\vdots & \ddots & \vdots & & \vdots \\
\delta_{i 1} & \cdots & \delta_{i j} & \cdots & \delta_{i n} \\
\vdots & & \vdots & \ddots & \vdots \\
\delta_{n 1} & \cdots & \delta_{n j} & \cdots & \delta_{n n}
\end{array}\right)\left[\begin{array}{c}
V_{1} \\
\vdots \\
V_{j} \\
\vdots \\
V_{n}
\end{array}\right]\right.
$$

Where: $|\mathbf{Y}|$ and $\boldsymbol{\delta}_{\mathrm{ij}}$ are the modulus and phase angle values of the dimension-reduced admittance matrix (5).

Finally, the linearized power flow equations are expressed as:

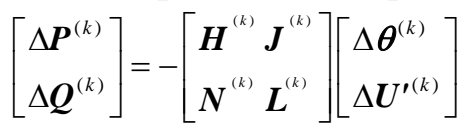

Similarly, the matrices for all Jacobian elements can be derived, the boldface terms all denote the matrices form.

$$
\begin{aligned}
& \left\{\begin{array}{l}
H_{i j}=\frac{\partial \Delta P_{i}}{\partial \theta_{j}}=-U_{i} U_{j} Y_{i j} \sin \left(\delta_{i j}\right) \\
H_{i i}=\frac{\partial \Delta P_{i}}{\partial \theta_{i}}=U_{i} \sum_{j \neq i} U_{j} Y_{i j} \sin \left(\delta_{i j}\right)-U_{i} U_{i} Y_{i i} \sin \left(\delta_{i i}\right) \\
\boldsymbol{H}=\operatorname{diag}(\mathbf{U}) * \operatorname{diag}\left[|\mathbf{Y}| \cdot * \sin \left(\boldsymbol{\delta}_{\mathbf{i j}}\right) *(\mathbf{U})\right]-\operatorname{diag}(\mathbf{U}) *\left[\mathbf{Y} \mid \cdot * \sin \left(\boldsymbol{\delta}_{\mathrm{ij}}\right)\right] * \operatorname{diag}(\mathbf{U})
\end{array}\right. \\
& \left\{\begin{array}{l}
N_{i j}=\frac{\partial \Delta P_{i}}{\partial U_{j}} U_{j}=-U_{i} U_{j} Y_{i j} \cos \left(\delta_{i j}\right) \\
N_{i i}=\frac{\partial \Delta P_{i}}{\partial U_{i}} U_{i}=-U_{i} \sum_{j \neq i} U_{j} Y_{i j} \cos \left(\delta_{i j}\right)-U_{i} U_{i} Y_{i i} \cos \left(\delta_{i i}\right) \\
\boldsymbol{N}=-\operatorname{diag}(\mathbf{U}) * \operatorname{diag}\left[|\mathbf{Y}| \cdot * \cos \left(\boldsymbol{\delta}_{\mathbf{i j}}\right) *(\mathbf{U})\right]-\operatorname{diag}(\mathbf{U}) *\left[|\mathbf{Y}| * \cos \left(\boldsymbol{\delta}_{\mathbf{i j}}\right)\right] * \operatorname{diag}(\mathbf{U})
\end{array}\right. \\
& \left\{\begin{array}{l}
J_{i j}=\frac{\partial \Delta Q_{i}}{\partial \theta_{j}}=U_{i} U_{j} Y_{i j} \cos \left(\delta_{i j}\right) \\
J_{i i}=\frac{\partial \Delta Q_{i}}{\partial \theta_{i}}=-U_{i} \sum_{j \neq i} U_{j} Y_{i j} \cos \left(\delta_{i j}\right)+U_{i} U_{i} Y_{i i} \cos \left(\delta_{i i}\right) \\
\boldsymbol{J}=-\operatorname{diag}(\mathbf{U}) * \operatorname{diag}\left[|\mathbf{Y}| * \cos \left(\boldsymbol{\delta}_{\mathbf{i j}}\right) *(\mathbf{U})\right]+\operatorname{diag}(\mathbf{U}) *\left[|\mathbf{Y}| * \cos \left(\boldsymbol{\delta}_{\mathbf{i j}}\right)\right] * \operatorname{diag}(\mathbf{U})
\end{array}\right.
\end{aligned}
$$




$$
\left\{\begin{array}{l}
L_{i j}=\frac{\partial \Delta Q_{i}}{\partial U_{j}} U_{j}=U_{i} U_{j} Y_{i j} \sin \left(\delta_{i j}\right) \\
L_{i i}=\frac{\partial \Delta Q_{i}}{\partial U_{i}} U_{i}=-U_{i} \sum_{j \neq i} U_{j} Y_{i j} \sin \left(\delta_{i j}\right)-U_{i} U_{i} Y_{i i} \sin \left(\delta_{i i}\right) \\
\mathbf{L}=-\operatorname{diag}(\mathbf{Y}) * \operatorname{diag}\left[|\mathbf{Y}| * * \sin \left(\boldsymbol{\delta}_{\mathrm{ij}}\right) *(\mathbf{U})\right]+\operatorname{diag}(\mathbf{U}) *\left[|\mathbf{Y}| \cdot * \sin \left(\boldsymbol{\delta}_{\mathbf{i j}}\right)\right] * \operatorname{diag}(\mathbf{U})
\end{array}\right.
$$

It can be seen from the power mismatch formula (13) and Jacobian matrices of (16) to (19) that, the element of the Jacobian matrix has similar features to traditional power-equation-voltagevariable type power flow calculation:

1) The dimension of the Jacobian matrix is $2(n-1)$, much less than that of the traditional type of $2\left(n+m_{1}-1\right)$, because active and reactive power of the IC nodes are zero, thereafter the ${ }^{2 m_{1}}$ power equations are eliminated from the new power flow model naturally;

2) If the mutual admittance between node ${ }^{i}$ and ${ }^{j}$ is zero, the corresponding element in the Jacobian matrix is zero, namely, the new type of Jacobian matrix is still sparse;

3) From two off-diagonal blocks of the Jacobian matrix are also symmetric, which could save computation time potentially as traditional power flow model;

4) The difference between the proposed new model and traditional power flow model is that, the admittance matrix has a reduced dimension. In the meantime, the reduced admittance matrix $\mathbf{Y}_{\text {reduced }}$ in Eqn. (5) includes an inverse calculation of ${ }^{m_{1}}$ dimension of $\mathbf{Y}_{11}$.The power flow computational efficiency will highly depend on both the ratio of IC nodes in the power system and the total number of nodes of the power system. Typical standard systems will be tested in next section to validate the effectiveness of the proposed model.

\section{Case studies}

The proposed new power flow model with the elimination of IC nodes are coded in Matlab software, and typical IEEE standard systems [6], and several large scale real world systems are tested on the proposed model.

\subsection{Initial settings for the proposed power flow model}

Typically in the traditional power flow model, the initial voltage value can be selected by empirical settings, such as flat starting with voltage magnitude as 1 p.u. and phase angle as zero degree, would be quite robust. As for the proposed new power flow model, it can be the same, then the Newton-Raphson iteration can be performed.

As for the PV type nodes, their voltage magnitude can be set as their pre-given values.

\subsection{Convergence performance of the new power flow model}

The maximum power mismatches in relationship with iteration numbers are plotted in Fig. 1. The convergence threshold is set as 10-8, and it can be seen that all test systems can converge in 4 to 6 steps, which proves the effectiveness of the proposed dimension reduced power flow model. 


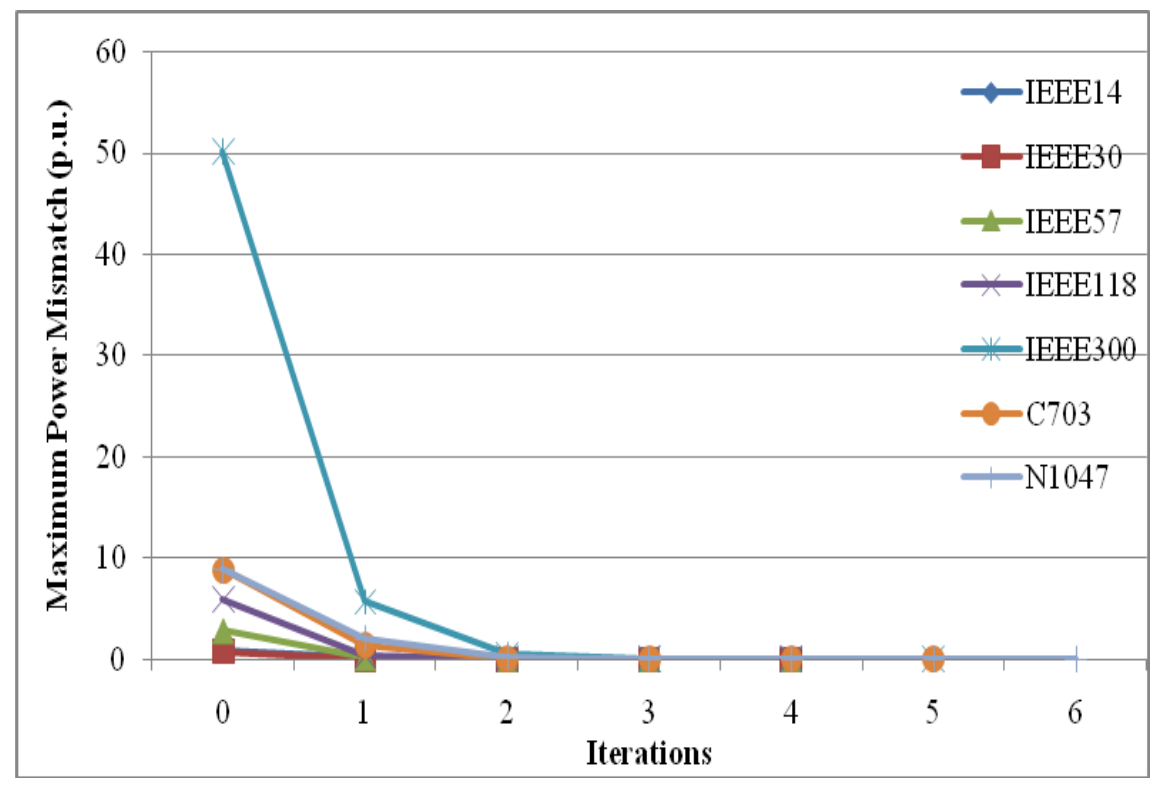

Fig. 1: Convergence curves of the new model based on Newton-Raphson method

\subsection{Comparison to traditional power flow model}

Further result in Table I shows the voltage magnitudes and phase angles of IEEE-14 system. It can be seen that the power flow results by the proposed model are the same to the traditional power flow model, according to the same initial condition and convergence criteria.

Table I: Convergence result of IEEE-14 bus systems

\begin{tabular}{ccccc}
\hline \multirow{2}{*}{$\begin{array}{c}\text { Node No. of } \\
\text { IEEE-14 }\end{array}$} & \multicolumn{2}{c}{ Traditional model } & \multicolumn{2}{c}{ New power flow model } \\
\cline { 2 - 5 } & $\begin{array}{c}\text { Voltage Magnitude } \\
\text { (p.u.) }\end{array}$ & $\begin{array}{c}\text { Phase Angle } \\
\left({ }^{\circ}\right)\end{array}$ & $\begin{array}{c}\text { Voltage Magnitude } \\
(\text { p.u.) }\end{array}$ & $\begin{array}{c}\text { Phase Angle } \\
\left({ }^{\circ}\right)\end{array}$ \\
\hline 1 & 1.06000000 & 0.00000000 & 1.06000000 & 0.00000000 \\
2 & 1.04500000 & -4.98258914 & 1.04500000 & -4.98258914 \\
3 & 1.01000000 & -12.72509994 & 1.01000000 & -12.72509994 \\
4 & 1.01767085 & -10.31290109 & 1.01767085 & -10.31290109 \\
5 & 1.01951386 & -8.77385390 & 1.01951386 & -8.77385390 \\
6 & 1.07000000 & -14.22094646 & 1.07000000 & -14.22094646 \\
7 & 1.06151953 & -13.35962737 & 1.06151953 & -13.35962737 \\
8 & 1.09000000 & -13.35962737 & 1.09000000 & -13.35962737 \\
9 & 1.05593172 & -14.93852130 & 1.05593172 & -14.93852130 \\
10 & 1.05098462 & -15.09728846 & 1.05098462 & -15.09728846 \\
11 & 1.05690652 & -14.79062203 & 1.05690652 & -14.79062203 \\
12 & 1.05518856 & -15.07558452 & 1.05518856 & -15.07558452 \\
13 & 1.05038171 & -15.15627634 & 1.05038171 & -15.15627634 \\
14 & 1.03552995 & -16.03364453 & 1.03552995 & -16.03364453 \\
\hline
\end{tabular}

In addition, Table II gives a quantitative analysis on the computational superiority of the proposed model, especially for large scale power systems with node number more than 300 . The calculation time for 703-bus and 1047-bus can be reduced by over $50 \%$, because the IC node ratio is actually high in large scale power systems. The computational effect is not time-saving enough for power systems with node number less than 118, mainly because the computation burden of inverse calculation of of $\mathbf{Y}_{11}$ during the admittance matrix dimension reduction. 
Table II: Computation efficiency comparison between the new model and traditional power flow model

\begin{tabular}{ccccc}
\hline $\begin{array}{c}\text { Node No. of test } \\
\text { systems }\end{array}$ & $\begin{array}{c}\text { No. of IC } \\
\text { nodes }\end{array}$ & $\begin{array}{c}\text { Node reduction } \\
\text { Ratio }\end{array}$ & $\begin{array}{c}\text { CPU Time for traditional } \\
\text { PF model }\end{array}$ & $\begin{array}{c}\text { CPU Time of New } \\
\text { model }\end{array}$ \\
\hline IEEE14 & 1 & $7.14 \%$ & 0.020804 & 0.039510 \\
IEEE 30 & 6 & $20.00 \%$ & 0.021347 & 0.042440 \\
IEEE 57 & 15 & $26.32 \%$ & 0.025704 & 0.048579 \\
IEEE 118 & 10 & $8.47 \%$ & 0.042031 & 0.063369 \\
IEEE 300 & 66 & $22.00 \%$ & 0.183164 & 0.157452 \\
C703 & 381 & $54.20 \%$ & 1.491008 & 0.519730 \\
N1047 & 540 & $51.58 \%$ & 3.289168 & 1.407905 \\
\hline
\end{tabular}

\section{Conclusions}

In modern large scale power systems, a large number of transmission substations are designed to deliver power to electricity users far away from power plants. And most of them are interconnecting (IC) nodes, which has the characteristic of zero power injection, thereafter causes zero current injection to the power system. A novel power flow calculation model is proposed, based on the elimination of IC node equations, to get more fast power flow calculation. The power network equations of IC nodes are eliminated so as to reduce the total dimension of the power flow model. Based on a deduction of power flow Jacobian in matrices forms, computational tests on IEEE standard systems and several real world large power systems are then performed to validate the proposed method, it is proved that the proposed method has excellent convergence performance as the traditional formulation of power flow model. Comparing to the traditional power flow model, the computation efficiency of our model is able to reduce the CPU time by over $50 \%$ for large scale power systems with node number more than 300 buses.

\section{Acknowledgements}

The research work was supported in part by China Postdoctoral Science Founda-tion under Grant 2013M542349, in part by the Fundamental Research Funds for the Central Universities of China under Grant xjj2013026, and in part by the State Key Laboratory of Electrical Insulation and Power Equipment under Grant EIPE14314.

\section{References}

[1] Saadat H. Power Systems Analysis. New York: McGraw-Hill, 2002.

[2] Tinney W F, Hart C E. Power Flow Solution by Newton's Method. Power Apparatus and Systems, IEEE Transactions on, 1967, PAS-86(11): 1449-1460.

[3] Stott B. Review of load-flow calculation methods. Proceedings of the IEEE, 62(7), pp.916-929, 1974.

[4] Jiang Q Y, Chiang H D, Guo C Xet al. Power-current hybrid rectangular formulation for interior-point optimal power flow[J]. Generation, Transmission \& Distribution, IET, 3(8), pp.748-756, 2009.

[5] Whei-Min L, Cong-Hui H, Tung-Sheng Z. A Hybrid Current-Power Optimal Power Flow Technique. Power Systems, IEEE Transactions on, 23(1), pp.177-185, 2008.

[6] Christie, Richard D. Power systems test case archive. Electrical Engineering dept., University of Washington, 2000. Available: http://www.ee.washington.edu/research/pstca/. 